\title{
Immediate bimaxillary full-arch reconstruction using an industrial outsourced digitally guided workflow - a feasibility demonstration
}

\author{
Adam S.C. Siu', James K.F. Chow ${ }^{2}$, Edward Hui', Mei M. Chong ${ }^{2}$, Michel Dard ${ }^{3,4}$ \\ ${ }^{1}$ Dental Implant and Maxillofacial Centre, Hong Kong, ${ }^{2}$ Dental Implant and Maxillofacial Centre, Hong Kong, P. R. China, \\ ${ }^{3}$ Department of Oral Diagnostic and Rehabilitation Sciences, College of Dental Medicine, Columbia University, New York, USA, \\ ${ }^{4}$ Straumann Group, Basel, Switzerland
}

\begin{abstract}
Bimaxillary immediate full-arch restorations can be prosthodontically, surgically, and esthetically demanding. State-of-the-art computer-assisted guided surgery can significantly improve the predictability and reliability of such procedures while reducing the chair time for the patient. This case report demonstrates how such procedures can be applied using an industrially outsourced service to assist implant planning and treatment preparation. A 63-year-old partially edentulous female with a failing dentition presented at our clinic. After medical assessment and the patient's request to avoid any edentulous episodes during treatment, a digitally guided bimaxillary full-arch reconstruction with an immediate restoration was performed within one session. The surgical and restorative procedures were performed as planned, allowing an immediate provisional restoration. The comparison of the planned and actual implant positions indicated slight inaccuracies between these positions. These deviations did not affect the immediate provisionalization, functionality, or esthetics of the immediate or final prosthetic rehabilitation. Fully digitally guided workflows represent a valuable tool for the efficient and reliable immediate transition from a failing dentition to a fixed reconstruction. The application of an outsourced and assisted digital planning service allows immediate access to digitally guided surgery and can improve efficiency. Deviations between planned and actual implant positions were most likely related to the rotational degrees of freedom around the teeth used for guide retention. (JOURNAL OF DENTAL IMPLANT RESEARCH 2021;40(4):121-133)
\end{abstract}

Key Words: Guided surgery, Immediate dental implant loading, Immediate placement, Digital

\section{INTRODUCTION}

The introduction of digital technologies has a tremendous impact on dentistry ${ }^{1)}$. With ongoing digitalization, whole subdisciplines like, e.g., implant dentistry and orthodontics can progressively be bridged and integrated into combined workflows ${ }^{2}$. One of the cornerstones of digital dentistry is acquiring, visualizing, and processing three-dimensional (3D) information of the patients' dental, osseous and soft tissue anatomy ${ }^{3)}$. A multitude of different techniques like cone-beam computer tomography
(CBCT), intraoral scanners (IOS), desktop scanners (DS), and even face scanners are today applied as part of routine digital treatment plans ${ }^{4}$.

Computer-guided template-based implant dentistry (guided surgery) offers the ability to plan the implantation procedure "backward" based on prosthetic demands and online visualize and validate the planned designs ${ }^{5,6)}$. The planning and execution of digital surgeries are mainly based on two sets of data: DICOM data (digital imaging and communication), which represent the patients' osseous and dental anatomy, and STL data

Received August 13, 2021, Revised September 15, 2021, Accepted September 22, 2021.

(c) Journal of Dental Implant Research.

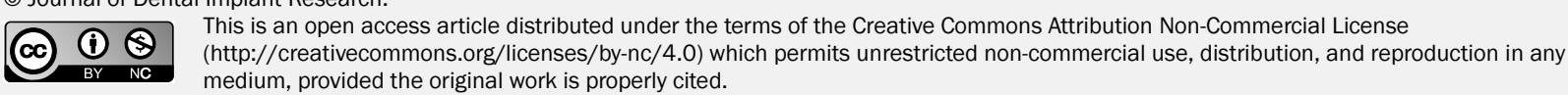

Correspondence to: Adam S.C. Siu

Dental Implant and Maxillofacial Centre, 1901-05, 12-13, 19/F The Center, 99 Queen's Road Central, Hong Kong, HKSAR, P.R.China. Tel: +852-2851-4888, Fax: +852-2851-4700, E-mail: dr.adamsiu@gmail.com

The authors would like to thank Dr. Albrecht Schnappauf of Dental Wings Inc. for providing the technical advice and support regarding coDiagnostiX ${ }^{\mathrm{TM}}$ and Novonexile AG (Switzerland) for writing assistance and editorial support in the preparation of the manuscript. 
(Standard Triangle Language), which include anatomical surface contour information of teeth and soft tissue. Both data sets can be obtained in one session using CBCT, IOS, or DS scanners ${ }^{7}$. Individual data sets need to be combined (registered) into one fully digitalized 3D anatomic model $^{8)}$. This model serves to plan the detailed implant restoration, clinical workflow, and CAD/CAM of surgical guides and provisional prosthesis for immediate restoration $^{9,10)}$.

The potential advantages and limitations of guided surgery over traditional, non-digitalized workflows are still subject to active discussions ${ }^{10,11)}$. Especially in more advanced and complex clinical procedures, guided surgery can significantly facilitate the diagnosis, treatment planning, and surgical procedures and render the whole treatment more predictable and efficient ${ }^{12,13)}$. Aesthetically demanding, high-risk, or procedures with complex prosthetic objectives like immediate restorations might significantly benefit from guided approaches ${ }^{14-17)}$. In daily practice, the implantation of digital technologies may be hindered by financial and time constraints. Further, a marked learning curve has been associated with such technologies ${ }^{18,19)}$. Recently, digital workflows that offer digital implant planning and delivery of guides and immediate provisionals have become available as part of an outsourced centralized service - Smile in a Box ${ }^{\mathrm{TM}}$. Such service may provide direct access to digital technologies without overcoming the mentioned hurdles related to their first-time access.

The present case report aimed to illustrate the detailed steps and critical elements of a bimaxillary full arch rehabilitation of a partially dentate patient using a complete digitally guided workflow as assisted by outsourced centralized services. Specifically, this case involved immediate digitally guided implant placement and immediate provisional restoration with a CAD/CAM prefabricated temporary prosthesis and a final restoration with a CAD/CAM fabricated screw-retained metal acrylic prosthesis. The detailed steps of this pilot partly outsourced workflow will be illustrated. Further, this pilot case evaluated implant placement accuracy and discussed possible influencing factors in the context of the contemporary literature.

\section{CASE REPORT}

\section{Initial patient conditions and medical history}

A 63-year old female presented in our clinics with ill-fitting upper and lower removable partial dentures and with a complaint of pain in her upper right premolars. A dental exam revealed residual maxillary dentition in positions 14,15 , and 27. Teeth 14 and 15 were overcrowned. Tooth 15 was affected by irreversible and acute pulpitis. Residual dentition in the mandible was present in positions 34 to 44 , with teeth 32 to 34 and 42 to 44 being overcrowned. Radiographic examination revealed periapical radiolucency at both abutment teeth of the upper and lower splinted crowns indicating a periodontal involvement. Assessment of the osseous anatomical conditions by CBCT revealed a Skeletal Class III relationship with a severe atrophic anterior maxilla. Further, a reduced occlusal vertical dimension with irregular occlusal plane caused by dentoalveolar compensation was identified. Abundant and thick keratinized soft tissues characterized the edentulous segments of the alveolar ridge. The initial dental and osseous conditions of the patient before treatment are illustrated in Fig. 1.

The patient presented good general health conditions, did not smoke, and was medicated for depression and osteoarthritis. No specific preconditions that would have excluded the patient from implant treatment were identified.

Various treatment options were discussed with the patient, including partial upper and lower removable restorations, upper implant-supported full-arch rehabilitation in conjunction with lower single implant rehabilitation after endodontic treatment, and bimaxillary fixed full-arch rehabilitation. The patient expressed a strong preference for an immediate functional bimaxillary fixed full-arch rehabilitation. The acute conditions of teeth 14 and 15 required immediate extraction and adaption of the upper conventional prosthesis before implant treatment.

\section{Treatment planning}

According to the patient's preference and the medical assessment, a complete bimaxillary reconstruction with an immediate provisional restoration was chosen. A 

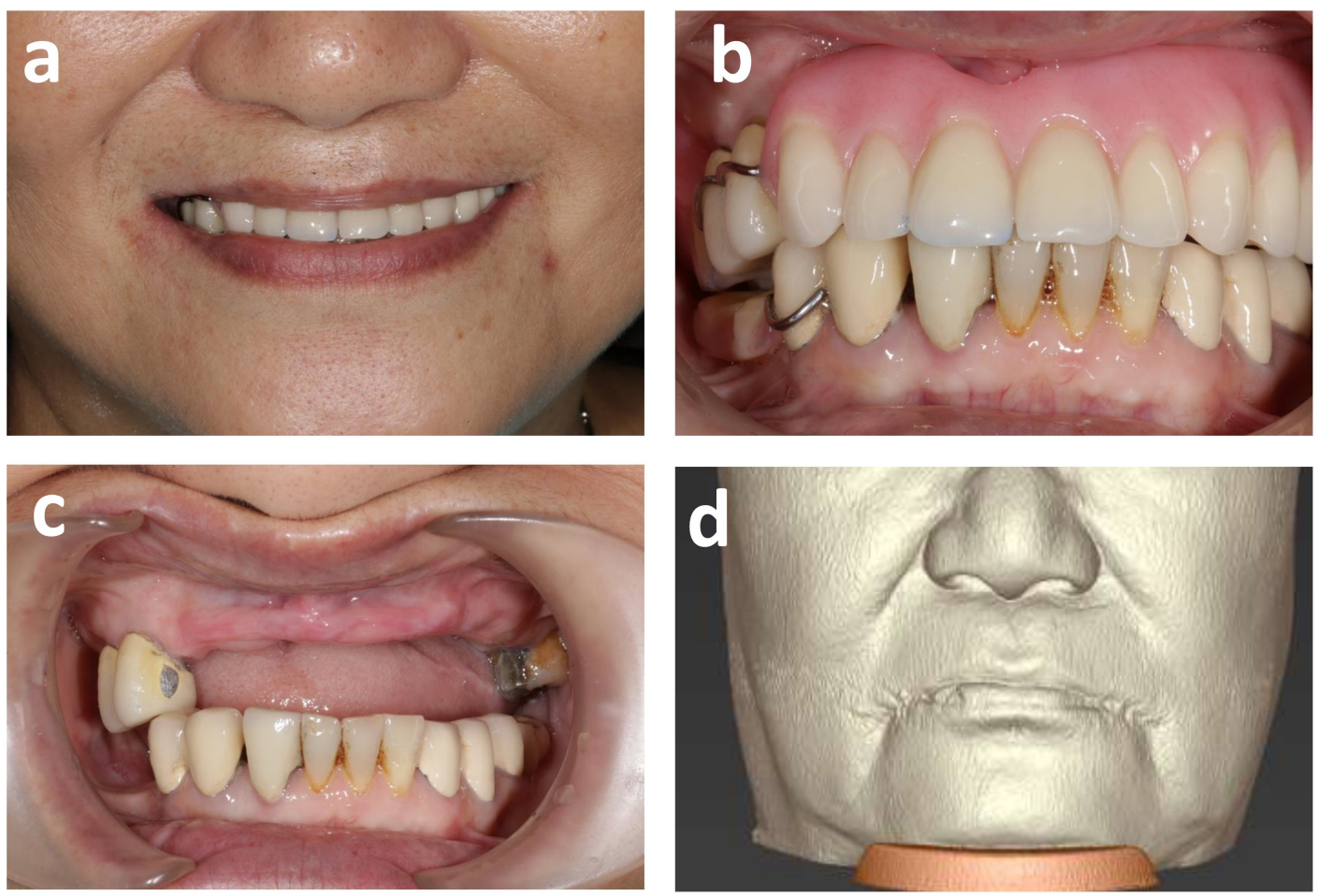

Fig. 1. Patient situation before treatment. (a) Frontal view before treatment. (b) Intraoral photograph of own dentition and conventional removable partial prosthesis before treatment. (c) Conditions of residual dentition prior to treatment. (d) 3D face scan of the patient prior to treatment.

Prosthodontist performed treatment planning. An Oral and Maxillofacial Surgeon verified implant positions and planning of the bone reduction. The treatment was designed as an entirely digitally guided workflow involving digital partly outsourced planning, guided implant placement, and immediate provisional prosthetic restoration within one single session.

Digital records for the treatment planning included 3D face scans (Vetra H1, Canfield Scientific Inc., New Jersey, USA) and dual CBCT scans (Newtom 5G, Newtom, Imola, Italy). Conventional full-arch impression and CBCT scans of both jaws were taken for diagnostic setup and planning of the new partial dentures at corrected occlusal vertical dimensions (OVD).

Prosthetic planning of the temporary and final restoration was carried out using DWOS (Dental Wings, Germany). The surgical workflow was digitally planned using coDiagnosti $X^{\mathrm{TM}}$ (Dental Wings, Germany). The presented case was performed with an industrially outsourced digital planning service called "Smile in a box" (SiaB, Institut Straumann AG, Switzerland). This pilot case describes the first time application of this service to a dental clinic (Dental implant and Maxillofacial Center, Hong Kong). In brief, the used service offered an assisted digital planning that was fully scalable from assisting up to providing complete digital planning. The service provided all necessary tools for the guided surgery and immediate restoration, including surgical guides and the temporary prosthesis. Additionally, a complete surgical set with all required surgical and prosthetic components, including implants, abutments, guides, drills, etc., was provided. The outsourced service and assisted planning allowed for an efficient procedure with reduced chair time for the practitioner and the patient. In the described case, data acquisition and prosthetic planning were performed in-house. The implant restoration, associated surgical workflows, guides, and provisional restoration were planned in conjunction with and manufactured by the SiaB team based on data sets provided by the clinic.

\section{Prosthetic planning and data integration of the virtual planning model}

Registering anatomical data of the patients' osseous, prosthodontic, and esthetic conditions into a 3D anatomi- 
cal planning model and segmenting such models to visualize and analyze specific anatomic aspects represent crucial steps during digital treatment planning. In the presented case, the corresponding DICOM and STL data sets were obtained from CBCT dual scans and desktop scans of master casts obtained from a conventional impression during one single session, respectively.

Fig. 2 illustrates the individual steps of data integration into the 3D planning model. First, a digital prosthetic wax-up based on desktop scans of the conventional mas- ter casts at corrected OVD (Fig. 2a) was designed using DWOS (Dental Wings, Germany) (Fig. 2b). CBCT DICOM, scan prosthesis STL files, and virtual wax-up datasets were imported into the implant planning software (coDiagnostiX (cDX), Dental Wings, Germany). Scan prostheses were modified with six fiducial markers along the vestibular rim to register the DICOM data sets from CBCT dual scans ${ }^{6}$. Fig. $2 \mathrm{c}$ to $2 \mathrm{f}$ illustrate the segmentation of CBCT data into maxilla and mandible, dental structures, and fiducial markers, respectively. The CBCT of the scan
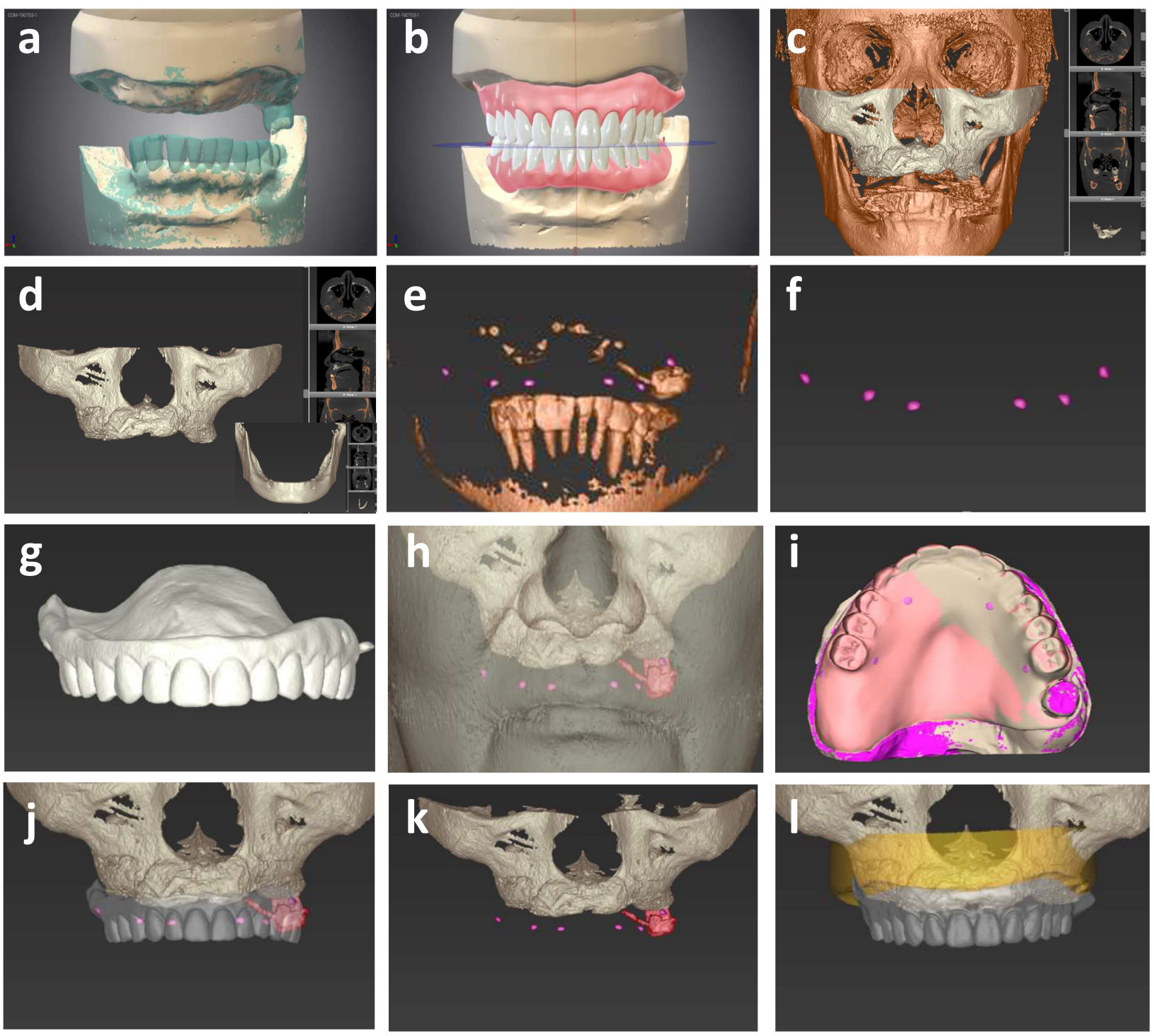

Fig. 2. Digital and prosthetic planning models. (a) Digital models of residual dentition from lab scans. (b) Digital wax-up of the planned prosthesis with corrected OVD. (c) CBCT scan and segmentation into (d) maxilla and mandible, (e) upper and lower teeth. (f) Fiducial markers. (g) Scan prosthesis. (h) Integrated model from face scan and CBCT scan segmented into maxilla, fiducial markers of scan prosthesis, and remaining upper molar. (i) Composite 3D image of scan prosthesis, plaster cast model, and fiduciary markers. (j) Integrated CBCT scan and STL model of scan prosthesis. (k) Maxilla with remaining teeth and fiducial markers. (I) Integrated planning model of CBCT scan and digital wax-up. 
prosthesis is shown in Fig. 2g. Next, CBCT data were aligned with face scan data (Fig. 2h) and were registered with the STL data sets of the scan prosthesis (Fig. 2i, 2j) using the fiducial markers (Fig. 2k) and with the virtual wax-up (Fig. 2l).

\section{Planning of implant restoration}

Implant types, positions, and angulation were planned based on the virtual planning models within a virtual case planning meeting between the Smile in a Box team, the surgeon, the restorative dentist, and the dental technician.

The planned maxillary and mandibular implant restorations based on the aligned digital wax-ups are illustrated in Fig. $3 \mathrm{a}$ to $3 \mathrm{c}$ and $3 \mathrm{~d}$ to $3 \mathrm{f}$, respectively. Specifically, in the maxilla, six equidistant axially oriented tapered implants (guided Bone Level Tapered (BLT) Institut Straumann AG, Switzerland) were planned in positions 16, 14, 12, 22, 24 and 26. This configuration allowed to support the first-molar-to-first-molar one-piece restoration without distal cantilevers. Planning was conducted to support immediate loading and under consideration of the moderate age of the patient. Adequate horizontal and vertical osseous dimensions, specifically in the distal maxillary aspect, allowed to realize the configuration without bone grafting ${ }^{20)}$.

The mandible displayed adequate horizontal and vertical dimensions in the anterior aspect and a relatively progressed atrophy in the distal aspects. Therefore, the mandibular first-molar-to-first-molar restoration was planned based on four implants in positions 35, 32, 42, and 45 . Distal implants were axially inclined to allow for an adequate $\mathrm{A} / \mathrm{P}$ spread $^{20)}$.

Implant diameters and lengths in the maxilla were as follows: mesial: $\varnothing 3.3 \times 12 \mathrm{~mm}$, mesiodistal: $\varnothing 4.1 \times 10 \mathrm{~mm}$, and distal: $\varnothing 4.8 \times 12 \mathrm{~mm}$ (Fig. 3a). Only one implant type (Bone Level Tapered (BLT), Ø4.1×12 mm, Institut Straumann AG, Switzerland) was chosen for the mandible. Posterior implants were planned angulated (Fig. 3d). Bone reduction was necessary to accommodate the subcrestal placement of mandibular implants (Fig. 3e). The mandibular crestal bone profile was planned based on the positions of the implant shoulder and is illustrated in Fig. 3f.

\section{Guide design and fabrication}

Based on the planned implant positions (maxilla and mandible) and the reduced crestal profiles (mandible), the
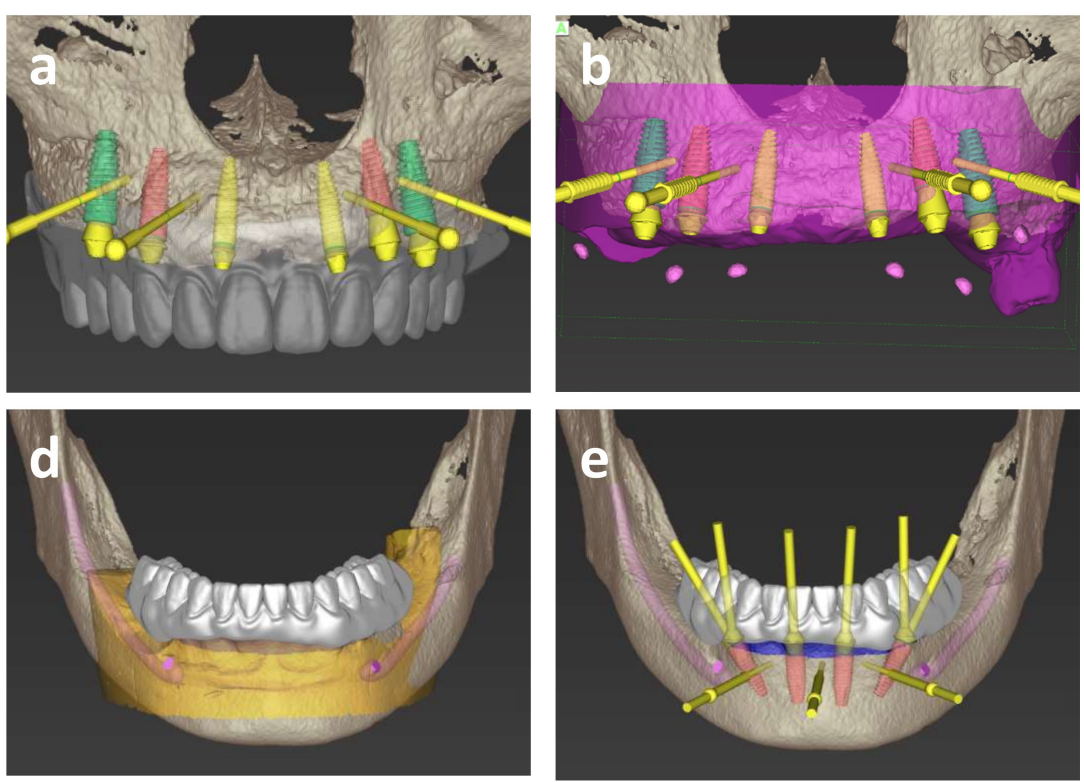
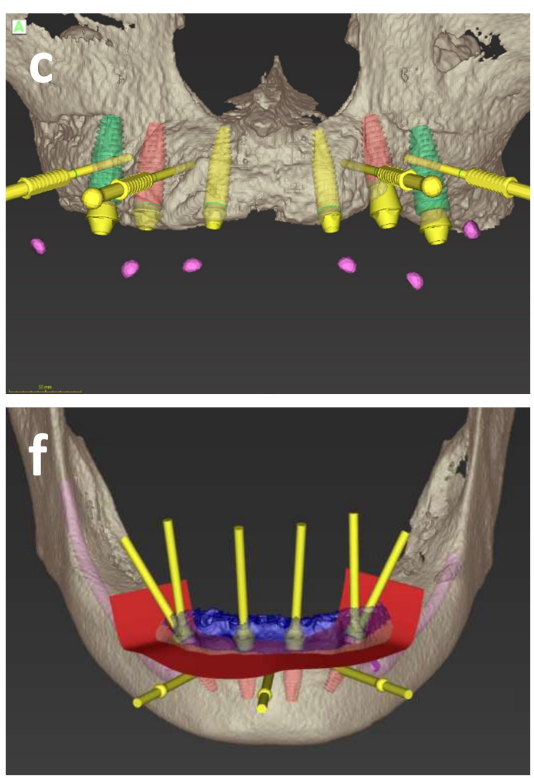

Fig. 3. 3D planning of the implant restoration in the maxilla (a to c) and mandible (d to f). (a) Maxilla with aligned digital wax-up, implant restoration, and anchoring pins. (b) Planning model with soft tissue contour (pink surface). (c) Fiduciary markers. (d) Mandible with aligned digital wax-up. (e) Implant restoration. f) Planning of bone resection (red plane) with blue coded section planned for resection. 


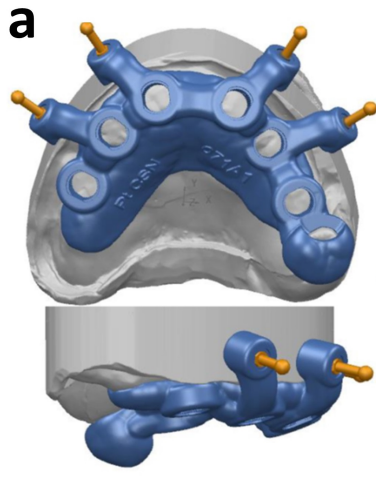

C
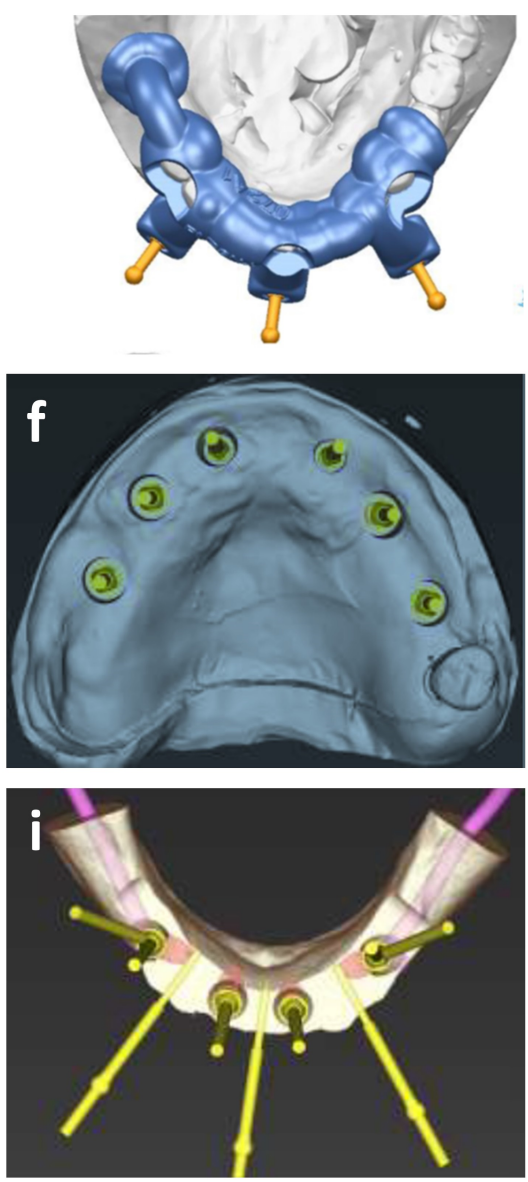

b
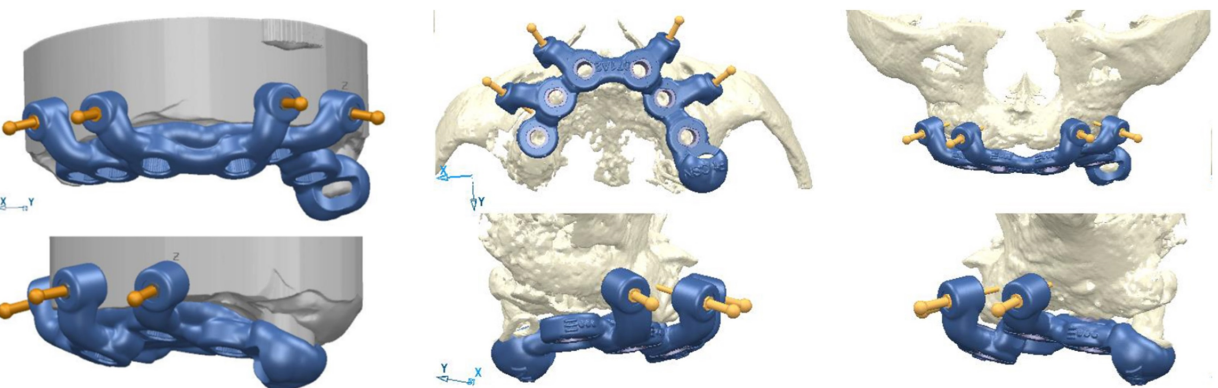

e
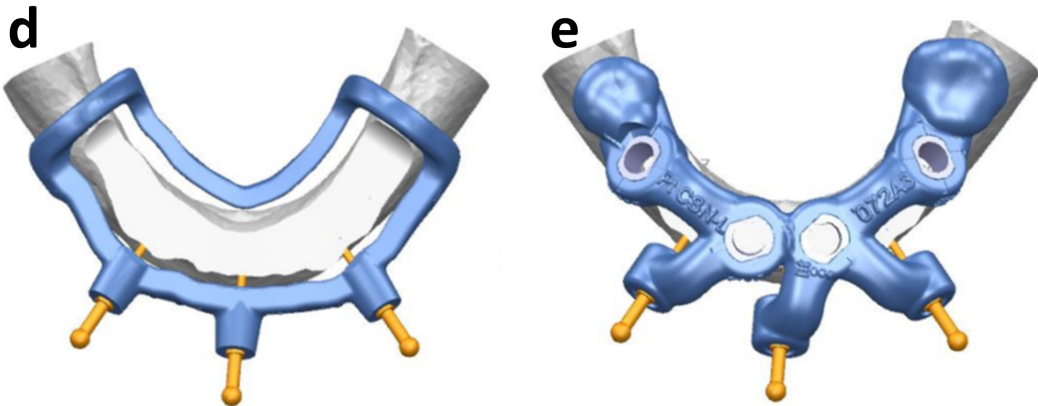

g
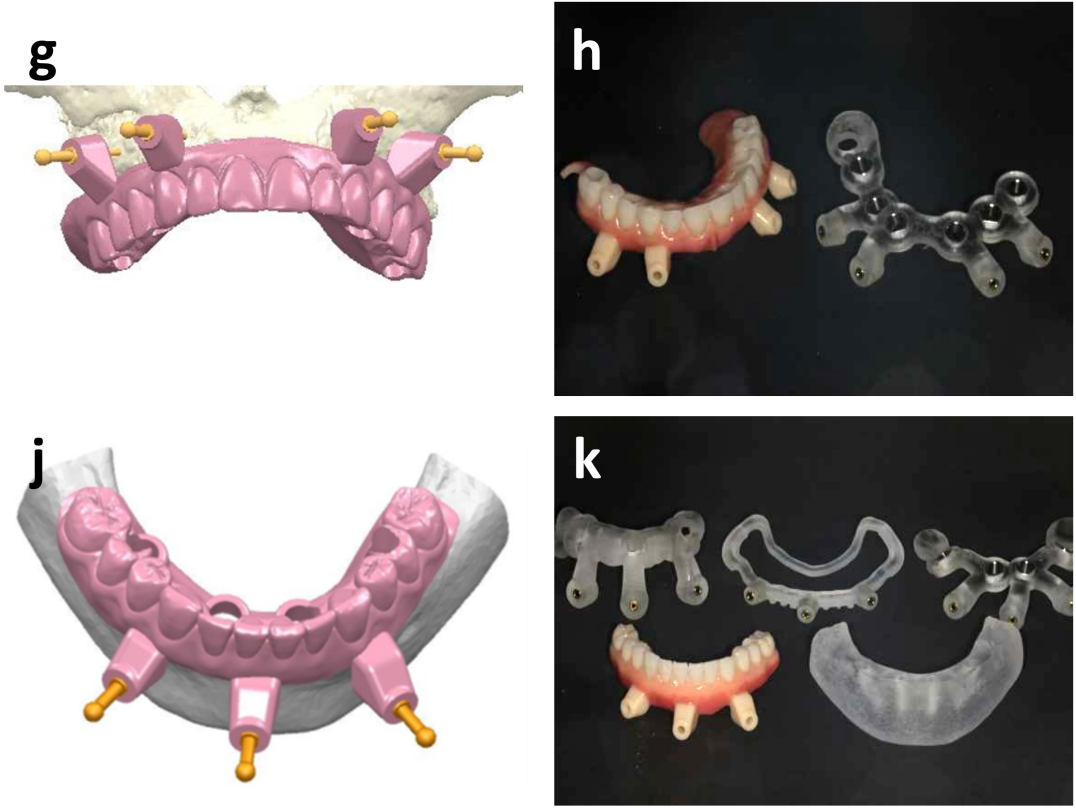

Fig. 4. 3D representation of planned maxillary $(a, b)$ and mandibular $(c \sim e)$ surgical guides (pinhole drill guide (a, d), surgical guide (b, e), and bone resection guide (d). (f) Maxillary DWOS soft tissue contour 3D anatomy model with implant positions. (g) 3D model of planned immediate maxillary provisional. (h) Manufactured immediate provisional and surgical drill guide. (i) Mandibular 3D bone reduced model with implant positions and anchoring pins. (j) 3D model of planned immediate mandibular provisional. (k) Mandibular surgical guides, anatomic jaw model, and immediate provisional.

positions of anchoring pins and the set of surgical guides were designed (Fig. 4). Specifically, 2 and 3 surgical guides were designed for the maxillary (Fig. 4a, 4b) and mandibular (Fig. 4c $\sim 4 \mathrm{e}$ ) procedures. The maxillary concept was based on two surgical guides: an anchoring pin guide (APG) and the surgical guide. The AGP was sup- ported by the palatal and alveolar gingiva and the residual upper left molar and designed to place four anchoring pins in positions 15, 13, 23, and 25 (Fig. 4a). The mandibular workflow included three surgical guides: a tooth retained APG for the placement of three anchoring pins in positions 34, 31, and 43 (Fig. 4c), a bone reduction 
guide (BRG) (Fig. 4d), and the surgical guide (Fig. 4e). All surgical guides had a sleeve height of $5 \mathrm{~mm}$ and were designed $4 \mathrm{~mm}$ above the bone crest.

The provisional prosthetic restorations were planned in DWOS (Fig. 4g, 4j) based on the digital wax-up and under consideration of the planned implant positions (Fig. 4f, 4i) (cDX, XOrder). Drill guides and prosthetic designs were exported from DWOS for centralized additive or subtractive manufacturing (Fig. 4h, 4k). The immediate provisional restorations (Smile in a Box, Straumann, Switzerland) were designed with vestibular flanges for pin anchoring to facilitate the pickup of the temporary copings from the screw-retained abutments (SRA) during provisionalization. Milled PMMA prefabricated provisional were surface veneered in the associated laboratory using pink resin composite for gingivae and composite stain for crowns.

\section{Surgical procedure}

Correct fit and function of all delivered surgical guides and instruments were validated before surgery as part of a dry run, which was carried out according to the guided surgery protocols using plaster cast models.

The surgical procedure was carried out under monitored anesthesia care (MAC) in combination with local infiltration anesthesia (Xylestesin-A with 2\% Lignocaine (3M ESPE)). Fig. 5 illustrates the guided surgical procedure in the mandible, which started with the APG-supported placement of 3 anchoring pins (Neodent Fixation Pin, Length $28 \mathrm{~mm}, \varnothing 1.4 \mathrm{~mm}$ ) in positions 34, 31, and 43. The correct position of the guide was verified inter-alia using the verification windows (Fig. 5a). After removing the APG, residual teeth were extracted, and a mucoperiosteal flap following mid-crest incision was extended over the area planned for bone reduction. The coronal aspect of the mandible was resected in horizontal buccolingual direction using a piezotome (Piezosurgery 3 (Mectron, Carasco, Italy)) at the level of the BRG (Fig. 5d to $5 \mathrm{f})$.

Four guided implants (guided BLT, Straumann, Switzerland) $(\varnothing 4.1 \times 12 \mathrm{~mm})$ were placed using the surgical guide (Fig. $5 \mathrm{~g}$ to $5 \mathrm{~h}$ ). Axial and apical guidance during osteotomy preparation was supported by guided drills and drill handles (Institut Straumann, Switzerland).
Mandibular implant osteotomies were completed after pilot drilling without implant bed fine preparation (Ø 3.5 $\mathrm{mm}$ ) (Fig. 5h). Implant placement depth was controlled using guided implants and corresponding stop keys (Fig. 5i). Healing abutments were mounted on the implants, followed by primary wound closure using 4/0 Vicryl (Ethicomn Inc., Johnson \& Johnson, USA).

The maxillary procedure involving six implants was carried out accordingly but without resection or reshaping the alveolar bone. Osteotomy preparation included thread predrilling to account for the higher portion of cortical bone. Implants were restored with screw-retained abutments (Straumann ${ }^{\circledR}$ screw-retained abutment, Straumann, Switzerland, 0 and $30^{\circ}$ angulation, gingival heights: $1 \mathrm{~mm}, 2.5 \mathrm{~mm}, 4 \mathrm{~mm}$ ) followed by primary wound closure. Subsequently, healing abutments in the mandible were replaced with screw-retained abutments before the immediate provisionalization.

\section{Immediate prosthetic restoration}

The immediate provisional restoration is illustrated in Fig. 6. In detail, temporary titanium copings were mounted on SRAs, and the provisional prosthesis was brought into position using the anchoring pins (Fig. 6a). Next, the immediate prosthesis was fixed on the titanium copings using cold-curing acrylic (Unifast Trad, GC America, USA) (Fig. 6b, 6c).

Following bite registration (Regisil Rigid, Dentsply Caulk, USA) (Fig. 6d), the prosthesis was picked up from the patient's mouth, implant analogs were attached and embedded in a plaster cast model, and the flanges of the prosthesis were removed (Fig. 6e).

After adjusting occlusal surfaces, the provisional prosthesis was installed back to the patient's mouth (Fig. 6f, $6 \mathrm{~g}$ ). As illustrated by the occlusal analysis after immediate provisionalization occlusal contacts were predominantly localized on the incisors (TScan, Tekscan, Inc., USA) (Fig. 6h). Occlusion was subsequently corrected and adapted chairside, and a soft splint prepared by deep draw forming was delivered for the initial healing period (Fig. 6i).

\section{Follow up and final restoration}

Fig. $7 \mathrm{a} \sim 7 \mathrm{~d}$ illustrates the occlusion with the immedi- 

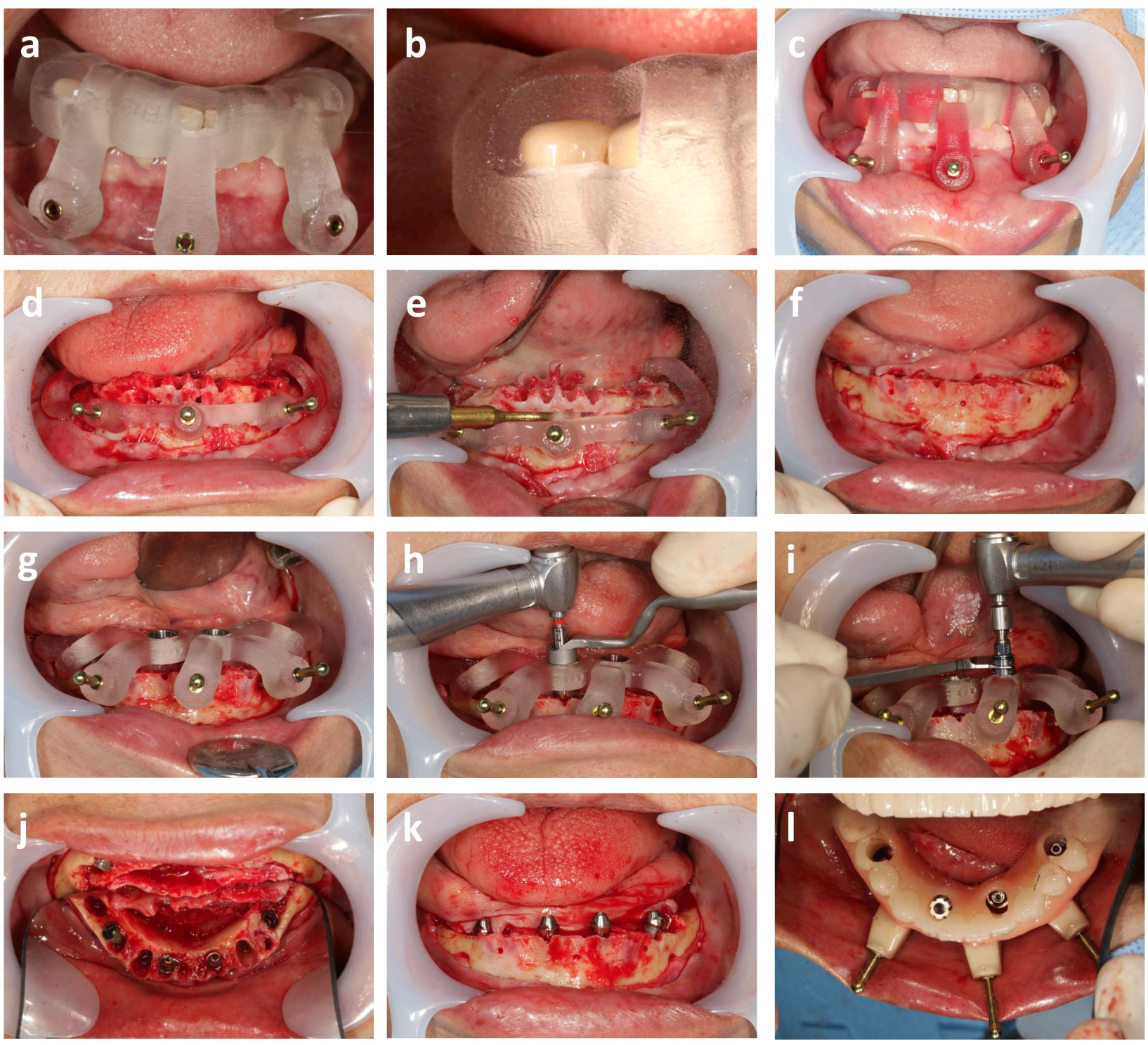

Fig. 5. Surgical procedure in the mandible. $(a \sim c)$ Placement of anchoring pins with pinhole drill guide. ( $d \sim f)$ Horizontal bone resection using a piezotome at the level of the bone reduction guide. (g) Surgical guide after placement. (h) Guided drilling using a guide handle for depth control. (i) Guided placement of tapered implant with stop key for depth control. (j) Occlusal view after implant placement. (k) Implants with screw-retained abutments before flap closure. h) Occlusal view on the mandibular prosthesis mounted on anchoring pins with Titanium coping in position 26.

ate provisional and the soft tissue situation after removal of the provisional and with temporary abutments at the 3 months follow up respectively. At this follow-up, an intermediate provisional with improved veneered esthetic was prepared for the patient using a conventional laboratory technique. Specifically, abutment level open tray impressions were taken after splinting screw-retained impression posts with a premanufactured $\mathrm{CoCr}$ bar suprastructure with flowable composite (Fig. 7e). Fig. $7 f$ and $7 \mathrm{~g}$ illustrate the resulting intermediate provisional re- storation on the plaster cast model and after delivery to the patient's mouth.

The final prosthesis consisted of a $\mathrm{CoCr}$ screw-retained bar (SRB) (Straumann, Switzerland) with a hybrid design (Teeth and denture base: Lucitone, Dentsply Sirona, USA) delivered 9 months post-surgery. The final prosthesis was based on working impressions from the intermediate provisional and new open tray impressions. Additionally, 3D face scans and face bow records were generated to plan and adjust the prosthetic midline, occlusal plane, and po- 

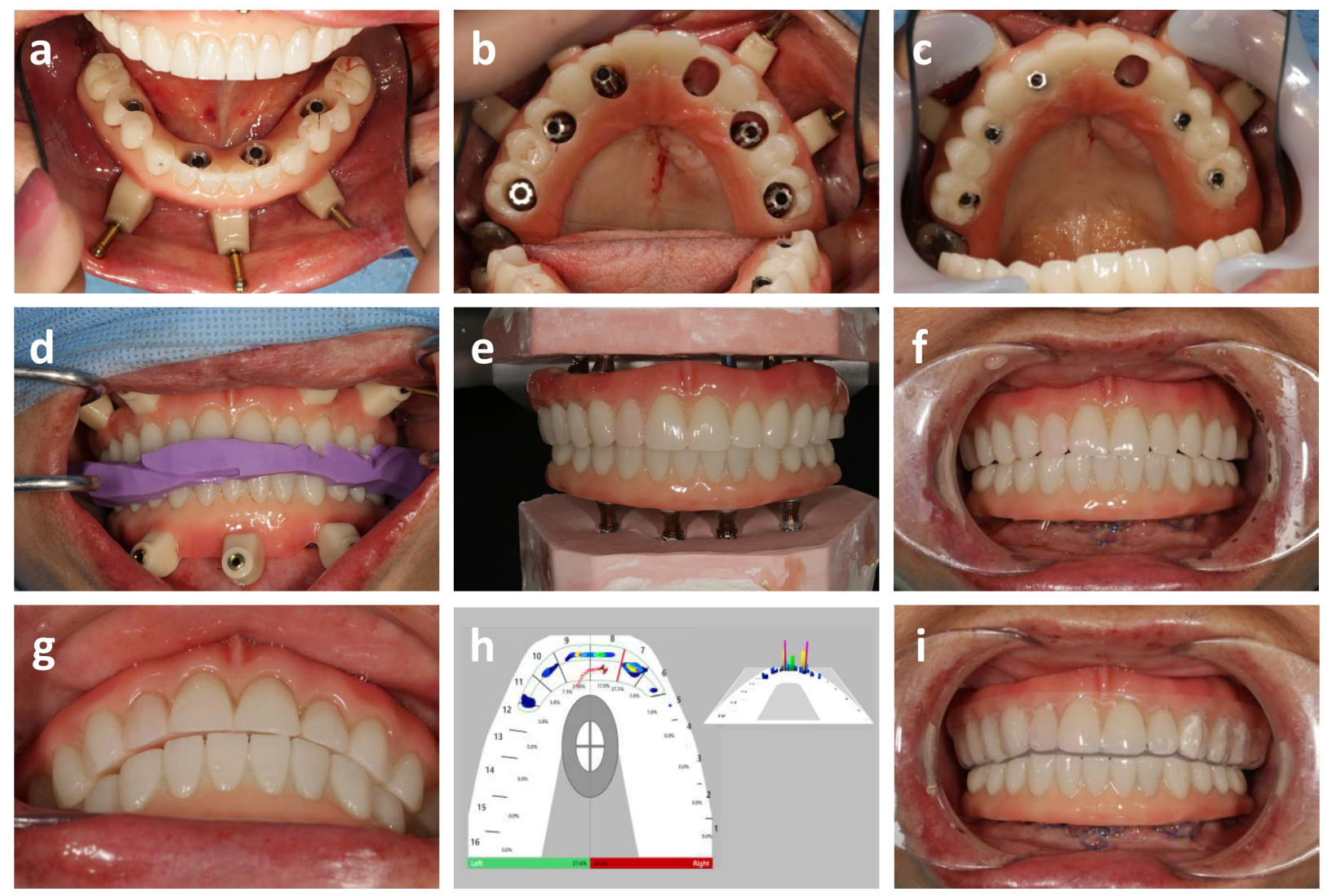

Fig. 6. Immediate provisional restoration. (a) Placement of provisional restoration on temporary copings using anchoring pins. (b) Occlusal view on the maxillary provisional before fixation and (c) after fixation on temporary copings. (d) Bite registration. (e) Pickup of implant positions on a master cast using abutment analogs. (f, g) Bite verification after occlusal adjustment. (h) Digital occlusal analysis (only upper jaw shown). (i) Immediate provisional with a soft splint.

sitional fine-tune the final prosthesis. The SRB final prosthesis was designed in DWOS using digital wax-ups, gingival models, and implant positions from previous models (Fig. 7h, 7i). The milled CoCr SRB intermediate was tried in the patient's mouth (Fig. 7k). Upper and lower open tray impressions were taken and used with jaw records to manufacture and align the final denture with the SRB (Fig. 7l). Fig. $7 \mathrm{~m}$ and $7 \mathrm{n}$ show the resulting final prosthetic restoration before and after delivery along with the 9 months post-op panoramic radiograph (Fig. 7o).

\section{DISCUSSION}

This case report demonstrates the capabilities of fully digital guided workflows to efficiently and precisely achieve the immediate bimaxillary restoration of a partially edentulous patient in one single session. The digitally planned and guided procedure did allow to immediately deliver the prefabricated provisional restoration and re- sulted in a predictable and acceptable occlusion. This indicates that the deviations between planned and actual implant positions in the mandible and maxilla had a sufficient level of accuracy. Residual inaccuracies could be well compensated during the final restoration.

The accuracy of implant placement represents one of the most critical criteria of guided surgery. Specifically, placement accuracy is essential for the integrity of critical anatomical structures and allows for a predictable and precise immediate provisionalization. Tahmaseb et al. and Schneider et al. have recently systematically reviewed the currently available literature regarding implant placement accuracy of guided procedures, Implant placement accuracy is usually analyzed in the form of four parameters, i.e., (1) spatial deviation at the entry point, (2) spatial deviation at the implant apex, (3) deviation in placement depth relative to the crestal bone level, and (4) deviation of the placement angle.

The ability to install the provisional prefabricated re- 

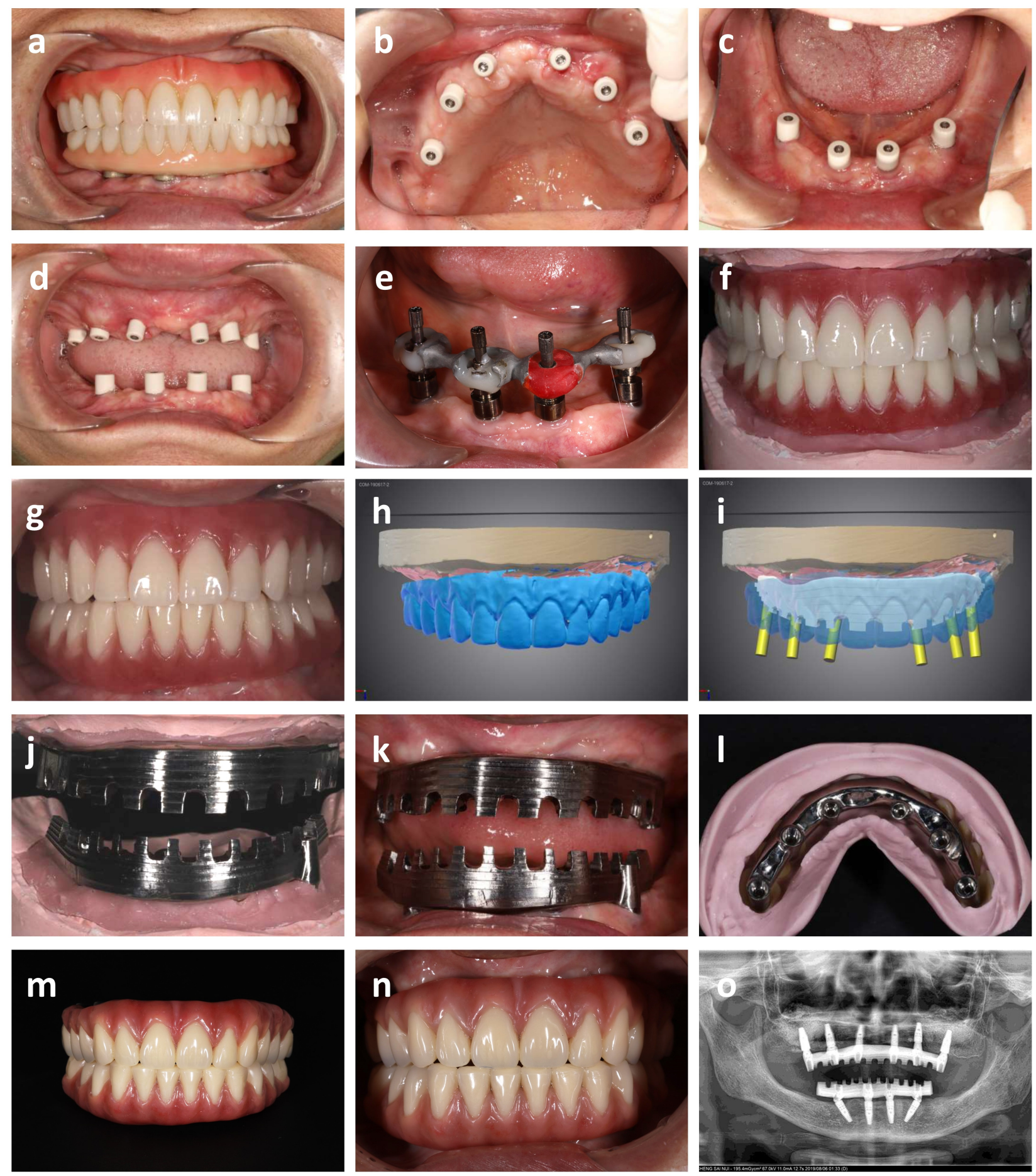

Fig. 7. Patient follow-up, intermediate and final restoration. (a) 3 months follow-up frontal view. (b $\sim$ d) Maxillary and mandibular occlusal and frontal views of soft tissue healing 3 months post-op. (e) Open tray impression with screw-retained impression posts splinted with $\mathrm{CoCr}$ Bar and fixed flowable composite. (f) Intermediate prosthesis with pink veneered esthetics. (g) Patient with mounted intermediate prosthesis. (h, i) Staircase bar design on master cast model scan and digital wax-up with implant positions (DWOS). (j, k) Milled Titanium screw-retained bars on the cast and at try-in. (I) Mounting of the milled bar onto acrylic denture. $(\mathrm{m}$ and $\mathrm{n})$ Final denture before and at try-in. (o) Radiograph of final restoration 9 months post-surgery. 
storation immediately and without significant adaptation indicates that overall spatial and angular placement accuracies remained within the required tolerances of the procedure. However, the implant placement accuracies can also be evaluated directly and quantitatively by comparing pre-and postoperative DICOM data sets that include the planned and actual implant positions. These more sophisticated comparisons can represent a valuable tool to derive statistical random errors and visually identify systematic errors resulting from practical aspects of the procedure.

A special module in the planning software (treatment evaluation tool coDiagnosti ${ }^{\mathrm{TM}}$ ) was used to analyze the performance of the outsourced workflow. Individual preand postoperative scans were matched using at least three characteristic and identifiable anatomical landmarks per jaw ${ }^{3}$. Fig. 8 shows the resulting color-coded overlays of the planned (blue) and actual (red) implant and anchoring pin positions in the maxilla (Fig. 8a) and mandible (Fig. 8b). From this qualitative comparison, distinct differences between the placement accuracies of implants and anchoring pins in the maxilla and mandible were identified. Specifically, the placement of anchoring pins and implants could be considered relatively accurate in the mandible. In contrast, deviations between the planned and actual implant and pin positions appeared more pronounced in the maxilla. In the maxilla, these deviations occurred to increase from the left distal to the right distal
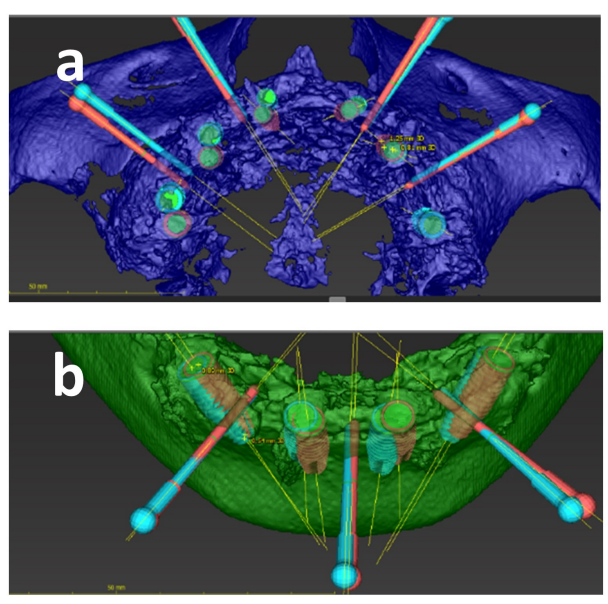

Fig. 8. Matched pre-and postoperative DICOM data sets in occlusal view of the maxilla (a) and mandible (b) comparing planned (blue) and real (red) positions of implants and anchoring pins (treatment evaluation tool, coDiagnosti ${ }^{\mathrm{TM}}$ ). position and reached apical deviations in the order of approximately one implant diameter in the right distal segments. In the mandible, mainly angular inaccuracies could be identified, which appeared to be most pronounced for the central implants.

Tahmaseb et al. have recently performed a metanalysis of published reports on implant placement accuracy of guided procedures. From their analysis of almost 2400 placed implants, the authors calculated a mean error at the entry point of $1.12 \mathrm{~mm}$ and an average angular deviation of $3.89^{\circ}$. From a qualitative perspective, the reported deviations are estimated to be well in line with the order of magnitude observed in this case report except for the implants placed in the right distal maxilla. These implants appeared to be displaced distally relative to planned entry points by approximately one implant diameter, indicating a systematic error resulting from the workflow and patient's specific procedural and anatomic aspects.

From a procedural aspect, we assume that the observed differences in the qualitative inaccuracies between the mandible and maxilla and between individual maxillary implants and pins might be related to differences in anatomic structures that supported the APG. This assumption is mainly supported by the fact that placement accuracies of maxillary implants and pins were similarly asymmetrically distributed. Specifically, when comparing the mandibular and maxillary procedures, the APG in the mandible was mainly supported by a relatively high number of anterior residual teeth. The posterior part of the mandibular APG was supported by the alveolar gingiva. By contrast, the corresponding APG in the maxilla was mainly palatal mucosa supported and further retained by only one remaining molar in the left quadrant in position 27. We, therefore, assume that the observed patterns of placement inaccuracy, i.e., angular inaccuracies of mainly anterior mandibular implants and spatial inaccuracies of the maxillary implants that were most pronounced in the right quadrant, might be related to rotational movements of the APG around the tooth retention during placement of the anchoring pins.

Few studies in the literature report on the accuracy of implant placement in the maxilla using gingiva supported or primarily gingiva supported surgical templates like they have been used in this case. Verhamme et al., 
e.g., have recently published a study involving 30 edentulous patients. The authors have reported a mean error of $1.59 \mathrm{~mm}$ and maximum errors of up to $4.21 \mathrm{~mm}$ for the entry point in such indications ${ }^{21}$. The latter value is more comparable to the deviations observed in the right maxillary segment analyzed in the here presented case. The authors associated these higher deviations with limited mouth opening and access problems to the anterior implantation site. Interestingly the authors have observed that these limitations often cause a shift of the guide in a mesial direction, while the implant positions in our case were shifted distally. Again, these specific differences might indicate that the deviation in distal direction observed herein might be well explained by the asymmetric distribution of mechanical support of the APG rather than other factors as described in the literature.

More generally, our observations suggest that the accuracy of individual surgical guided protocols might be improved by carefully considering the local anatomic conditions that might affect the residual degrees of freedom of the surgical guides. Treatment sequences might need to take such effects into account to render any guided procedures accurate and precise. Also, the qualitative analysis of implant deviations related to the procedural aspects of guided surgery can help to identify any potential source for placement accuracy.

\section{CONCLUSION}

In this case report, we have illustrated the capabilities of guided surgery using a fully digital workflow to perform bimaxillary immediate full arch restorations in one session. The case demonstrates in detail the individual steps of a guided procedure. The application of an industrially outsourced planning workflow allowed for convenient and seamless access to an assisted digital workflow and the immediate conversion of a failing dentition into a fixed full-arch restoration. Possible sources for implant placement inaccuracies were likely associated with rotational movements of the anchoring pin guides around retention teeth.

\section{REFERENCES}

1. van Noort R. The future of dental devices is digital. Dent Mater 2012;28:3-12.

2. Kugel G, Ganz SD, Agarwal T. Digital technologies: a roundtable discussion on changing the face of dentistry. Dent Today 2017;36:18,20,22,24.

3. Verstreken K, Van Cleynenbreugel J, Marchal G, Naert I, Suetens $P$, van Steenberghe D. Computer-assisted planning of oral implant surgery: a three-dimensional approach. Int J Oral Maxillofac Implants 1996;11:806-10.

4. Mangano C, Luongo F, Migliario M, Mortellaro C, Mangano FG. Combining intraoral scans, cone beam computed tomography and face scans: the virtual patient. J Craniofac Surg 2018;29:2241-6.

5. D'haese J, Ackhurst J, Wismeijer D, De Bruyn H, Tahmaseb A. Current state of the art of computer-guided implant surgery. Periodontol 2000 2017;73:121-33.

6. Katsoulis J, Pazera P, Mericske-Stern R. Prosthetically driven, computer-guided implant planning for the edentulous maxilla: a model study. Clin Implant Dent Relat Res 2009;11:238-45.

7. Lanis A, Alvarez del Canto 0 . The combination of digital surface scanners and cone beam computed tomography technology for guided implant surgery using 3Shape implant studio software: a case history report. Int J Prosthodont 2015;28:169-78.

8. Christ A, Heister R, Anderl R. Reverse Engineering for the Utilization of Product Model Data for CAx Workflows in Dental Technology. In: 20th European Concurrent Engineering Conference, ECEC'2014 and the 10th Future Business Technology Conference, FUBUTEC'2014. Bruges, Belgium: EUROSIS-ETI, 2014 [cited 2019 Nov 30] ; 47-54.

9. Schubert O, Schweiger J, Stimmelmayr M, Nold E, Güth JF. Digital implant planning and guided implant surgery - workflow and reliability. Br Dent J 2019;226:101-8.

10. Wismeijer D, Joda T, Flügge T, Fokas G, Tahmaseb A, Bechelli D, et al. Group 5 ITI consensus report: digital technologies. Clin Oral Impl Res 2018;29:436-42.

11. Schneider D, Marquardt P, Zwahlen M, Jung RE. A systematic review on the accuracy and the clinical outcome of computer-guided template-based implant dentistry. Clinical Oral Implants Research 2009;20:73-86.

12. Colombo M, Mangano C, Mijiritsky E, Krebs M, Hauschild U, Fortin T. Clinical applications and effectiveness of guided implant surgery: a critical review based on randomized controlled trials. BMC Oral Health 2017;17:150.

13. Hultin M, Svensson KG, Trulsson M. Clinical advantages of computer-guided implant placement: a systematic review. Clin Oral Implants Res 2012;23:124-35.

14. Pyo SW, Lim YJ, Koo KT, Lee J. Methods used to assess the 3D accuracy of dental implant positions in computer-guided implant placement: a review. J Clin Med 2019 Jan 7; 8. doi: 10.3390/jcm8010054

15. Scherer MD, McGlumphy EA, Seghi RR, Campagni WV. Comparison of retention and stability of implant-retained overdentures based upon implant number and distribution. Int J 
Oral Maxillofac Implants 2013;28:1619-28.

16. Bidra AS. Three-Dimensional Esthetic Analysis in Treatment Planning for Implant-Supported Fixed Prosthesis in the Edentulous Maxilla: Review of the Esthetics Literature: THREE-DIMENSIONAL ESTHETIC ANALYSIS. Journal of Esthetic and Restorative Dentistry 2011;23:219-36.

17. Nickenig H-J, Eitner S. Reliability of implant placement after virtual planning of implant positions using cone beam CT data and surgical (guide) templates. Journal of Cranio-Maxillofacial Surgery 2007;35:207-11.

18. Al Yafi F, Camenisch B, Al-Sabbagh M. Is Digital Guided Implant Surgery Accurate and Reliable? Dental Clinics of North America 2019;63:381-97.
19. Tahmaseb A, Wismeijer D, Coucke W, Derksen W. Computer technology applications in surgical implant dentistry: a systematic review. Int J Oral Maxillofac Implants 2014;29:25-42.

20. Daudt Polido W, Aghaloo T, Emmett TW, Taylor TD, Morton D. Number of implants placed for complete-arch fixed prostheses: A systematic review and meta-analysis. Clin Oral Impl Res 2018;29:154-83.

21. Verhamme LM, Meijer GJ, Boumans T, de Haan AFJ, Bergé SJ, Maal TJJ. A clinically relevant accuracy study of computer-planned implant placement in the edentulous maxilla using mucosa-supported surgical templates: clinically relevant implant validation of computer-planned implants. Clinical Implant Dentistry and Related Research 2015;17:343-52. 